\title{
Monkeys pass the mirror test after training
}

\author{
ZHOU Wen ${ }^{1,3^{*}} \&$ JIANG Yi ${ }^{2,3 *}$ \\ ${ }^{1}$ Key Laboratory of Mental Health, Institute of Psychology, Chinese Academy of Sciences, Beijing 100101, China; \\ ${ }^{2}$ State Key Laboratory of Brain and Cognitive Science, Institute of Psychology, Chinese Academy of Sciences, Beijing 100101, China; \\ ${ }^{3}$ CAS Center for Excellence in Brain Science, Institute of Psychology, Chinese Academy of Sciences, Beijing 100101, China
}

Received February 12, 2015; accepted February 14, 2015; published online March 5, 2015

Citation: Zhou W, Jiang Y. Monkeys pass the mirror test after training. Sci China Life Sci, 2015, 58: 405-406, doi: 10.1007/s11427-015-4830-7

There is no experiencing without an experiencer, the self. Whether the experiencer knows it is "the self" that is experiencing is a different and tricky philosophical issue. Dating back to Ivan Pavlov's dogs, the "experience" of animals has been the subject of scientific research for over a century, yet to which extent different animals possess the sense of self is still hotly debated today. From human's perspective, the sense of self is the corner stone of feelings, thoughts, and social interactions - a necessity of higher intelligence.

In 1970, Gordon Gallup Jr. [1] developed the mirror test, also known as the mark test, to determine whether a non-human animal has the ability of self-recognition. The test typically goes as follows: An animal is familiarized with a mirror. Then it is anaesthetized (or sent to sleep) and is marked with an odorless dye on the face. When it awakes, it is presented with the mirror again. If it touches the marked area on its face, which the animal cannot see except from the mirror, the animal is believed to have recognized itself. Over the past 45 years, many different species have been challenged with this deceptively simple test. Only a handful of them have passed it, including chimpanzees, orangutans, dolphins and elephants - animals that we usually think of as smart [2,3]. Humans can pass the test by the age of two [4]. Macaque monkeys, known for their intricate social structure and hierarchy, nonetheless fail the test. Is there an impassable line between macaques and great apes like chimpanzees? Is self-recognition, like the pass/fail nature of the mirror test, an all/none phenomenon?

*Corresponding author (email: zhouw@psych.ac.cn; yijiang@psych.ac.cn)
Maybe not. Self-recognition, and self-awareness in general, may have to do with experience and learning, as Gong and his colleagues showed in a recent paper published in Current Biology [5]. The macaque monkeys in their study went through visual-somatosensory training in front of a mirror before being given the mirror test. Each day of training began with a high-power laser light projecting to random locations on either side of the monkeys' face, which caused apparent irritation and naturally made them to touch the projected area. Then the high-power laser was replaced with a low-power laser of the same color that did not cause irritation. The monkeys could only see the projected area from the mirror. A touch of the projected area was rewarded with food. After two to five weeks of training, the monkeys learned to touch the area on their face that was marked by a non-irritant light spot or odorless dye but was not associated with somatosensory input. Then, without any food reward, they were given the actual mirror test. As it turned out, all seven trained monkeys passed the mirror test on the training chair. Five of them also passed it in their home cage. They even spontaneously utilized the mirror to explore the normally hidden parts of their body. That is to say, they acquired through training the knowledge that their reflections in the mirror were not others but themselves, i.e., self-recognition.

The findings by Gong and colleagues suggest that self-recognition may be a product of contingency learning rather than some sort of innate mental faculty. They also raise the possibility that training may help people with defi- 
cits in self-recognition and self-awareness. It is worth noting, however, that repetitive training in their study likely increased the behavioral relevance of the mark used in the mirror test and effectively directed the monkeys' attention to that mark. After all, something with its resemblance was associated with food during training. In this light, one could argue that the mirror test is not the ultimate test for self-recognition [3,6] - an animal with self-recognition may fail the mirror test simply because it does not find the mark meaningful enough. But then the argument goes on as to what constitutes meaning for any living creature.
1 Gallup GG Jr. Chimpanzees: self-recognition. Science, 1970, 167: 86-87

2 Gallup GG Jr. Self-awareness and the evolution of social intelligence. Behav Processes, 1998, 42: 239-247

3 Suddendorf T, Butler DL. The nature of visual self-recognition. Trends Cogn Sci, 2013, 17: 121-127

4 Amsteldam BK. Mirror self-image reactions before age two. Dev Psychobiol, 1972, 5: 297-305

5 Chang L, Fang Q, Zhang S, Poo MM, Gong N. Mirror-induced self-directed behaviors in rhesus monkeys after visual-somatosensory training. Curr Biol, 2015, 25: 212-217

6 Gallup GG Jr., Platek SM, Spaulding KN. The nature of visual self-recognition revisited. Trends Cogn Sci, 2014, 18: 57-58

Open Access This article is distributed under the terms of the Creative Commons Attribution License which permits any use, distribution, and reproduction in any medium, provided the original author(s) and source are credited. 